\title{
Photometry and models of selected main belt asteroids I. 52 Europa, 115 Thyra, and 382 Dodona
}

\author{
T. Michałowski ${ }^{1}$, T. Kwiatkowski ${ }^{1}$, M. Kaasalainen ${ }^{2}$, W. Pych ${ }^{3}$, A. Kryszczyńska ${ }^{1}$, P. A. Dybczyński ${ }^{1}$, \\ F. P. Velichko ${ }^{4,9}$, A. Erikson ${ }^{5}$, P. Denchev ${ }^{6}$, S. Fauvaud ${ }^{7}$, and Gy. M. Szabó ${ }^{8}$ \\ 1 Astronomical Observatory, Adam Mickiewicz University, Słoneczna 36, 60-286 Poznań, Poland \\ 2 Rolf Nevanlinna Institute, University of Helsinki PO Box 4, 00014 Helsinki, Finland \\ 3 Copernicus Astronomical Center, Bartycka 18, 00-716 Warszawa, Poland \\ ${ }^{4}$ Research Institute of Astronomy, Kharkiv Karazin National University, Sums'ka 35, 61022 Kharkiv, Ukraine \\ 5 Institute of Space Sensor Technology and Planetary Exploration, Rutherford str. 2, 12489 Berlin, Germany \\ 6 Institute of Astronomy, Rozhen National Observatory, PO Box 136, 4700 Smolyan, Bulgaria \\ 7 Astroqueyras Association, Mairie, 05350 Saint Veran, France \\ ${ }^{8}$ Department of Experimental Physics \& Astronomical Observatory, University of Szeged, 6720 Szeged, Dóm tér 9, Hungary \\ 9 Isaac Newton Institute of Chile, Crimean Branch
}

Received 12 June 2003 / Accepted 24 November 2003

\begin{abstract}
Photometric observations for 52 Europa (1995, 1997, 1999, 2000), 115 Thyra (1995, 1996, 1998, 2000), and 382 Dodona $(1996,1998,1999,2001)$ carried out at seven observatories are presented. Using all available lightcurves, the spin vectors, senses of rotation, and shape models of these three asteroids have been determined or refined.
\end{abstract}

Key words. techniques: photometric - minor planets, asteroids

\section{Introduction}

Current physical studies of asteroids follow two paths: one is a detailed investigation of selected objects, for which many methods of observation have been used, and the other focuses on deriving more basic characteristis of a large sample of asteroids so that their population could be studied from the statistical point of view. For the latter approach, photometric observations from the Earth are still one of the basic techniques. They are easy to do and enable determination of rotational periods, orientations of spin vectors, shape models, and physical properties of surfaces of asteroids.

The photometric database consists of the lightcurves of about 1000 asteroids, but spin vectors have been determined for only about 100 of them. Magnusson et al. (1989) provided a review of all techniques used in order to obtain poles and shapes of asteroids. One approach to obtain this information is to study the brightness variation af asteroids as they spin about their axes. Such a method, described by Michałowski (1993), has been used in the present work. We have also used the second method, so called lightcurve inversion described by Kaasalainen \& Torppa (2001) and Kaasalainen et al. (2001, 2003). Both methods are briefly presented in Sect. 4.

The present work is the first paper in a series devoted to enlargement of the number of asteroids with known physical

Send offprint requests to: T. Michałowski,

e-mail: tmich@amu.edu.pl parameters. For many asteroids lightcurves from one or two oppositions have been known and it would be enough to observe them at one or two more apparitions. The new photometric observations, combined with previously published ones, will be used to determine sidereal periods, coordinates of poles and triaxial ellipsoid models for the observed asteroids. These new models will be included into the existing database of spin parameters and used for statistical investigation of the collision evolution of the asteroids (cf. Paolicchi et al. 2003). The most extensive spin and shape database is the one compiled by Per Magnusson, and recently updated at the Poznan Astronomical Observatory, which can be found at http://www . astro.amu.edu.pl/Science/Asteroids/

\section{Observations and data reduction at Borowiec Observing Station}

As many of the Main Belt asteroids are quite bright during opposition, they can be observed photometrically with the help of small aperture telescopes. To determine asteroid lightcurve it is enough to cover its rotational period with about 50-100 points (Harris \& Lupishko 1989). Most of asteroid periods lie in the interval 6-12 hours, so to obtain a sufficient number of points per lightcurve, each measurement of brightness should last no longer than 3-6 min. This in turn means photometry with a $S / N=100$ can be done with a $0.5 \mathrm{~m}$ telescope, equipped with a typical front-side illuminated CCD. 
Most of our observations were carried out at the Borowiec Station of Poznań Astronomical Observatory, Poland. A $0.4 \mathrm{~m}, F / 4.5$ Newton reflector was used, equipped with a $K A F 400 \mathrm{CCD}$ camera and a set of Bessel $B V R I$ filters. An additional, clear glass filter was also used, so that unfiltered exposures could be mixed with the filtered ones without the need to refocus the optical system.

It soon appeared that to maintain low noise photomeric measurements, a clear filter (hereafter $C$ filter) should be used most of the time. As the front-illuminated, KAF400 CCD has the spectral response favouring red part of the spectrum, $C$ magnitudes can be treated as non-standard, broad band red magnitudes (Henden 2000). As most of asteroids display very small colour variations within their rotations, our $C$ lightcurves can be safely used together with other $R$ magnitude lightcurves.

A typical observing run started with bias, dark and twilight flat-field frames. If the weather at twilight was bad and no dusk or dawn flat-fileds could be obtained, frames from other nights were used. In such cases, however, flat-fields taken before and after that night were compared to make sure no chages in the optical system occured in the mean time.

Even if most exposures were made through the $C$ filter, each night additional $R$ and $V$ exposures were taken to allow subsequent measurements of colour indices of an asteroid and comparison stars. In order to determine transformation from the instrumental to the standard system, Landolt stars were observed at various air masses. It soon appeared, however, that extinction seldom remained constant throughout the night and displayed slight dependence on the azimuth. This is caused by an unfavourable local astroclimate influenced by a nearby lake. On the other hand, differential photometry at this site appeared to be quite good, with statistical noise in the brightness of the constant magnitude comparison stars being sometimes as low as 0.003 mag.

Times of the middle of exposures were taken from the acquisition computer, whose clock was adjusted at the beginning and end of the night with the help of the Frankfurt long wave radio signals. Thus the overall accuracy of the timing of observations was never worse than $0.5 \mathrm{~s}$.

CCD frames were reduced with the $S T A R L I N K$ package. Corrections for bias, dark current and flat-filed were applied and the aperture photometry was used to get the instrumental brightness of an asteroid, comparison and check stars. Aperture radii were usually set to $3 \cdot F W H M$, where $F W H M$ on a typical night amounted to $4^{\prime \prime}$.

Finally, results of the relative photometry of asteroids were transformed into the format of the electronic version of the Asteroid Photometric Catalogue which includes additional data necessary for succesful analysis of the lightcurves. The data were further analysed by making composite lightcurves and fitting them with a Fourier harmonics. These operations were performed by a software written at Poznań Observatory according to algorithms described in Magnusson \& Lagerkvist (1990). As a result, peak-to-peak amplitudes and epochs of the extrema were derived for composite lightcurves. These parameters were used for obtaining physical models of asteroids, presented in this paper.

\section{Observations of three Main Belt asteroids}

Photometric observations of three asteroids (52 Europa, 115 Thyra, and 382 Dodona) from 49 nights in the years 1995-2001 were made at seven observatories. The majority of the data (35 nights) came from Borowiec as mentioned above. The other observations were also carried out at Ostrowik (Warsaw University Observatory, Poland), Kharkiv (Kharkiv University, Ukraine), European Southern Observatory, Rozhen (Institute of Astronomy, Bulgaria), d'Anjou Observatory (Association Astronomique d'Anjou, France), and Szeged (University of Szeged, Hungary).

At Ostrowik, a $0.60 \mathrm{~m}$ Cassegrain telescope, equipped with a TEK512 CB CCD camera, was used (Udalski \& Pych 1992). CCD frames, collected through the $R$ Cousins filter were reduced with standard IRAF procedures and the profile photometry was obtained with the Daophot-II package.

A $0.70 \mathrm{~m}$ telescope with CCD camera and $V$ filter were used at Kharkiv Observatory. The frames were reduced with the synthetic aperture photometry package (ASTPHOT) developed at $D L R$ by Stefano Mottola (Mottola et al. 1995).

The asteroid 115 Thyra was also observed at ESO in 1995. These observations were performed with a $D L R$ camera at the Bochum telescope and reduced with the software developed by Stefano Mottola (Erikson et al. 2000).

In September 1997, the asteroid 52 Europa was observed at three observatories. A $0.60 \mathrm{~m}$ Cassegrain telescope equipped with a single-channel photometer was used at the Rhozen Observatory (Bulgaria). A transformation to the $U B V$ standard system has been carried out with standard algorithms (Denchev et al. 1998). Unfiltered photometry was carried out at the University of Szeged (Hungary) using a $0.28 \mathrm{~m}$ telescope and SBIG ST-6 CCD-camera. These observations were referred to the JATE asteroid survey (more details in Szabò et al. 2001 and references therein). At the Association Astronomique d'Anjou Observatory (located at Saint-Saturninsur-Loire, France) a $0.40 \mathrm{~m}$ Newton telescope and Hi-SIS22 CCD camera (KAF-400 chip) equiped with a Cousins $R$ filter were used. An aperture photometry of comparison stars and the asteroid was performed after standard correction for for bias, dark and flat-field.

From all the lightcurves, only the ESO, Kharkiv, and Rhozen photoelectric photometry data were transformed to the standard system as has been stated above. The rest of the observations have not been transformed, mainly because of nonphotometric weather condition and/or because the observing systems were equipped with only one standard filter.

Table 1 contains the aspect data for the asteroids observed. The first column is the date of the observation referring to the mid-time of the lightcurve observed. The next two columns are the distances (in astronomical units) from the asteroid to the Sun and the Earth, respectively. Column 4 is the solar phase angle, and Cols. 5 and 6 give the $J 2000.0$ ecliptic longitude $(\lambda)$ and latitude $(\beta)$, respectively, referred to the time in the first column. The names of the observatories are listed in the last column of the table.

The basic parameters of the asteroids are summarized in Table 2. Their IRAS diameters $(D)$, albedos, and taxonomic 
Table 1. Aspect data.

\begin{tabular}{|c|c|c|c|c|c|c|}
\hline \multirow[t]{2}{*}{ Date (UT) } & \multirow{2}{*}{$\begin{array}{c}r \\
(\mathrm{AU})\end{array}$} & \multirow{2}{*}{$\begin{array}{c}\Delta \\
(\mathrm{AU})\end{array}$} & \multirow{2}{*}{$\begin{array}{c}\text { Phase } \\
\text { angle } \\
\left({ }^{\circ}\right)\end{array}$} & \multicolumn{2}{|c|}{$\begin{array}{ll}\lambda & \beta \\
(\mathrm{J} 2000)\end{array}$} & \multirow[t]{2}{*}{ Obs. } \\
\hline & & & & $\left({ }^{\circ}\right)$ & $\left({ }^{\circ}\right)$ & \\
\hline \multicolumn{7}{|l|}{52 Europa } \\
\hline 19950529.9 & 3.207 & 2.346 & 11.21 & 211.34 & 10.18 & Ost \\
\hline 19950530.9 & 3.208 & 2.355 & 11.45 & 211.24 & 10.14 & Ost \\
\hline 19950531.9 & 3.209 & 2.364 & 11.70 & 211.14 & 10.10 & Ost \\
\hline 19970909.0 & 3.230 & 2.232 & 2.98 & 352.70 & -7.22 & Roz, Szg \\
\hline 19970926.1 & 3.215 & 2.238 & 4.81 & 349.30 & -7.56 & Anj \\
\hline 19990210.8 & 2.801 & 2.292 & 19.21 & 72.69 & -5.53 & Bor \\
\hline 19990228.9 & 2.795 & 2.523 & 20.66 & 75.20 & -4.54 & Bor \\
\hline 19990318.8 & 2.791 & 2.760 & 20.65 & 79.18 & -3.70 & Bor \\
\hline 19990319.8 & 2.791 & 2.773 & 20.61 & 79.43 & -3.65 & Bor \\
\hline 19990326.8 & 2.790 & 2.864 & 20.27 & 81.32 & -3.36 & Bor \\
\hline 19990330.8 & 2.789 & 2.915 & 20.01 & 82.47 & -3.21 & Bor \\
\hline 19990331.8 & 2.789 & 2.928 & 19.93 & 82.78 & -3.17 & Bor \\
\hline 20000215.0 & 2.921 & 2.083 & 12.14 & 183.53 & 7.10 & Bor \\
\hline 20000324.0 & 2.955 & 1.971 & 3.71 & 176.76 & 8.62 & Bor \\
\hline 20000421.0 & 2.982 & 2.138 & 12.42 & 172.31 & 8.62 & Bor \\
\hline \multicolumn{7}{|l|}{115 Thyra } \\
\hline 19950323.3 & 2.837 & 2.186 & 17.44 & 239.45 & -15.09 & ESO \\
\hline 19950425.2 & 2.837 & 1.903 & 9.25 & 235.59 & -17.21 & ESO \\
\hline 19950805.0 & 2.790 & 2.535 & 21.27 & 225.92 & -11.27 & ESO \\
\hline 19961014.8 & 2.025 & 1.193 & 20.35 & 338.90 & 15.19 & Ost \\
\hline 19961015.8 & 2.023 & 1.199 & 20.72 & 338.85 & 15.18 & Ost \\
\hline 19961227.7 & 1.940 & 1.840 & 30.00 & 356.64 & 11.98 & Kha \\
\hline 19961228.7 & 1.939 & 1.850 & 29.96 & 357.09 & 11.93 & Kha \\
\hline 19980310.9 & 2.576 & 1.598 & 4.74 & 169.85 & -12.38 & Bor \\
\hline 19980323.9 & 2.599 & 1.643 & 7.83 & 166.53 & -12.83 & Bor \\
\hline 19980324.9 & 2.601 & 1.649 & 8.18 & 166.29 & -12.84 & Bor \\
\hline 19980325.9 & 2.603 & 1.654 & 8.54 & 166.06 & -12.86 & Bor \\
\hline 19980326.9 & 2.604 & 1.660 & 8.89 & 165.84 & -12.87 & Bor \\
\hline 19980328.9 & 2.608 & 1.673 & 9.59 & 165.41 & -12.89 & Bor \\
\hline 19980329.9 & 2.609 & 1.679 & 9.95 & 165.20 & -12.89 & Bor \\
\hline 19980414.9 & 2.636 & 1.816 & 15.19 & 162.72 & -12.73 & Bor \\
\hline 19980416.9 & 2.639 & 1.837 & 15.75 & 162.53 & -12.68 & Bor \\
\hline 19980420.8 & 2.645 & 1.880 & 16.79 & 162.24 & -12.58 & Bor \\
\hline 19980423.8 & 2.650 & 1.914 & 17.50 & 162.10 & -12.49 & Bor \\
\hline 19980425.9 & 2.653 & 1.937 & 17.95 & 162.04 & -12.42 & Bor \\
\hline 19980427.9 & 2.656 & 1.961 & 18.37 & 162.01 & -12.36 & Bor \\
\hline 19980430.9 & 2.660 & 1.998 & 18.95 & 162.02 & -12.25 & Bor \\
\hline 20001024.1 & 1.923 & 1.068 & 20.39 & 68.73 & 20.86 & Bor \\
\hline 20001102.1 & 1.925 & 1.024 & 17.03 & 67.77 & 21.49 & Bor \\
\hline 20001103.1 & 1.926 & 1.020 & 16.65 & 67.62 & 21.54 & Bor \\
\hline \multicolumn{7}{|l|}{382 Dodona } \\
\hline 19961015.0 & 3.614 & 2.628 & 2.72 & 24.36 & 9.61 & Ost \\
\hline 19961016.0 & 3.615 & 2.629 & 2.66 & 24.17 & 9.61 & Ost \\
\hline 19980123.9 & 3.501 & 2.809 & 12.82 & 71.91 & 6.98 & Bor, K \\
\hline 19980129.8 & 3.494 & 2.874 & 13.87 & 71.66 & 6.73 & Bor \\
\hline 19980201.8 & 3.491 & 2.910 & 14.33 & 71.60 & 6.60 & Bor \\
\hline 19990120.2 & 2.941 & 2.082 & 11.15 & 154.91 & -1.84 & Bor \\
\hline 19990122.1 & 2.938 & 2.064 & 10.56 & 154.69 & -1.93 & Bor \\
\hline 19990317.9 & 2.844 & 1.955 & 10.85 & 144.51 & -3.99 & Bor \\
\hline 20010827.0 & 3.372 & 2.431 & 7.38 & 358.37 & 6.28 & Bor \\
\hline 20010920.0 & 3.405 & 2.407 & 2.26 & 353.77 & 6.91 & Bor \\
\hline
\end{tabular}

Observatory Code: Ost - Ostrowik; Roz - Rozhen; Szg - Szeged; Anj - Anjou; Bor - Borowiec; ESO; Kha - Kharkiv.
Table 2. Asteroid parameters.

\begin{tabular}{lccc}
\hline \hline Asteroid & $D(\mathrm{~km})$ & albedo & Type \\
\hline 52 Europa & 303 & 0.058 & $\mathrm{C}$ \\
115 Thyra & 80 & 0.275 & $\mathrm{~S}$ \\
382 Dodona & 58 & 0.161 & $\mathrm{M}$ \\
\hline
\end{tabular}

types are taken from The Small Bodies Node of the NASA Planetary Data System (http://pdssbn. astro.umd.edu/).

The results of our observations are presented in Figs. 1-13 as composite lightcurves. They have been obtained with a procedure described by Magnusson \& Lagerkvist (1990). The lightcurves have been composited with the synodical periods shown in the graphs. Points from different nights are marked with different symbols. The vertical position of each individual lightcurve is obtained to minimize the dispersion of data points relative to their neighbours. The abscissae are the rotational phases with the zero points corrected for light-time. When the observational data are reduced to the standard system, we can present the lightcurve with $V(1, \alpha)$ magnitude reduced to the unit distances to the Earth and Sun and given phase angle $\alpha$ (see Figs. 2, 5, 7 and 11). If it is necessary, the magnitude offsets for each night are also given in these figures.

\subsection{Europa}

The first photometric observations of 52 Europa were performed during five nights in November-December 1976 (Scaltriti \& Zappala 1977). Within the period of 5.631 hours, the asteroid showed a lightcurve with a small amplitude of $0.09 \mathrm{mag}$ and one pair of extrema. Observations from January-February 1983 (Zappala et al. 1983) revealed the lightcurve with the amplitude of 0.10 mag and two well defined maxima and minima during the period mentioned above. The observations from two nights in April 1984 (Barucci et al. 1986) showed again one pair of extrema and small amplitude of 0.08 mag. In 1986 (Dotto et al. 1995) 52 Europa showed the quasi-sinusoidal lightcurve with much larger amplitude of 0.23 mag. Michałowski et al. (1995) observed this asteroid during two other apparitions: October-November 1992 and February 1994. The lightcurves had two pairs of extrema and amplitudes of 0.10 and $0.11 \mathrm{mag}$, respectively. In September-October 1997 (Lopez-Gonzalez \& Rodriguez 1999) Europa was observed again and the quasi-sinusoidal lightcurve had the amplitude of $0.20 \mathrm{mag}$.

We observed 52 Europa on 15 nights during four apparitions: 1995, 1997, 1999, and 2000. All these observations confirmed the previously estimated period of 5.631 hours.

The observational data obtained on three consecutive nights in May 1995 covered only about $80 \%$ of rotational cycle (Fig. 1) but the overall shape of the lightcurve was visible. It was very asymmetrical with two maxima at different levels. The whole amplitude seems to be 0.16 mag.

The lightcurve from two nights in September 1997 (Fig. 2) had a quasi-sinusoidal shape with an amplitude of $0.20 \mathrm{mag}$. These data were in good agrement with those obtained 


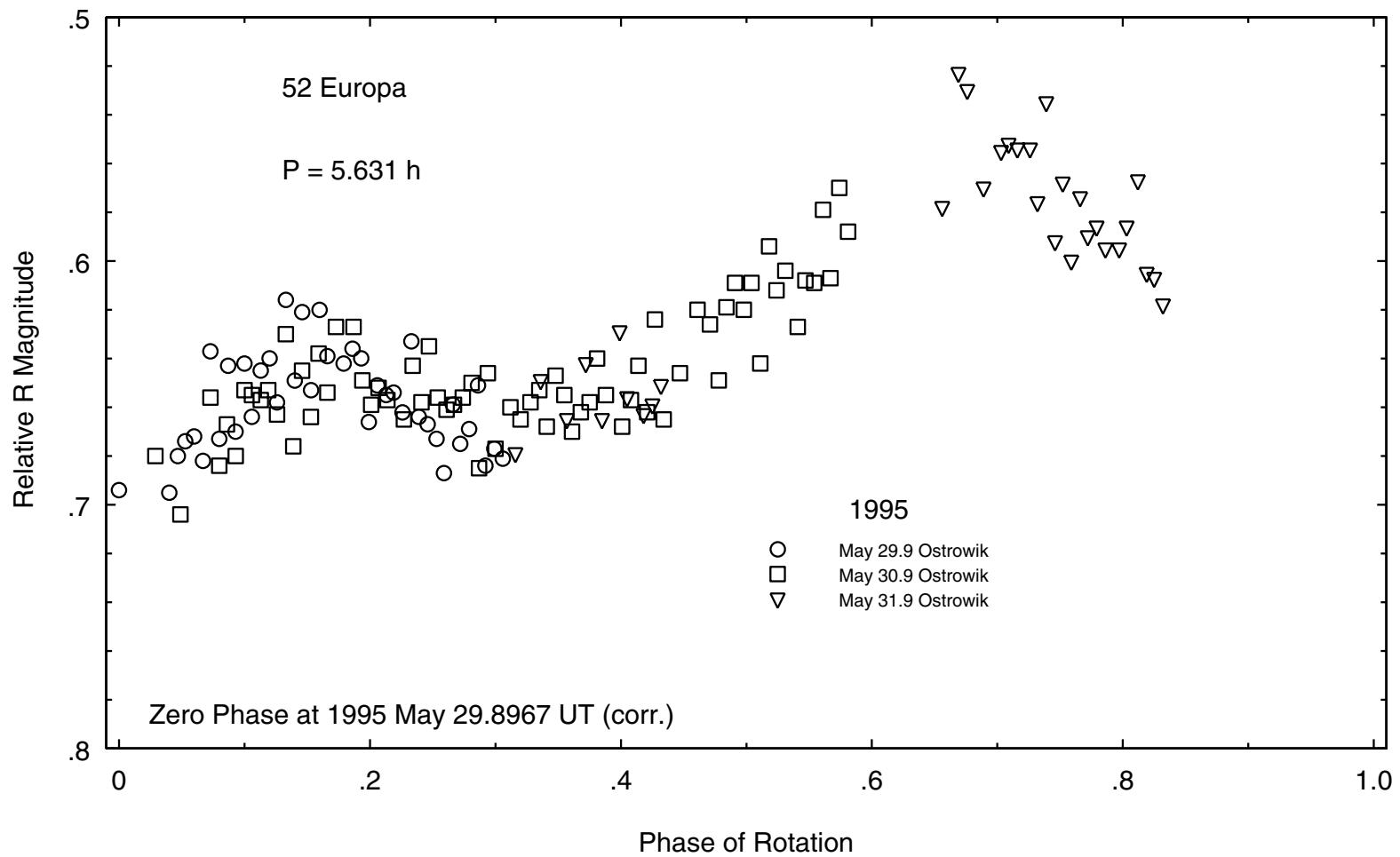

Fig. 1. Lightcurve of 52 Europa in 1995.

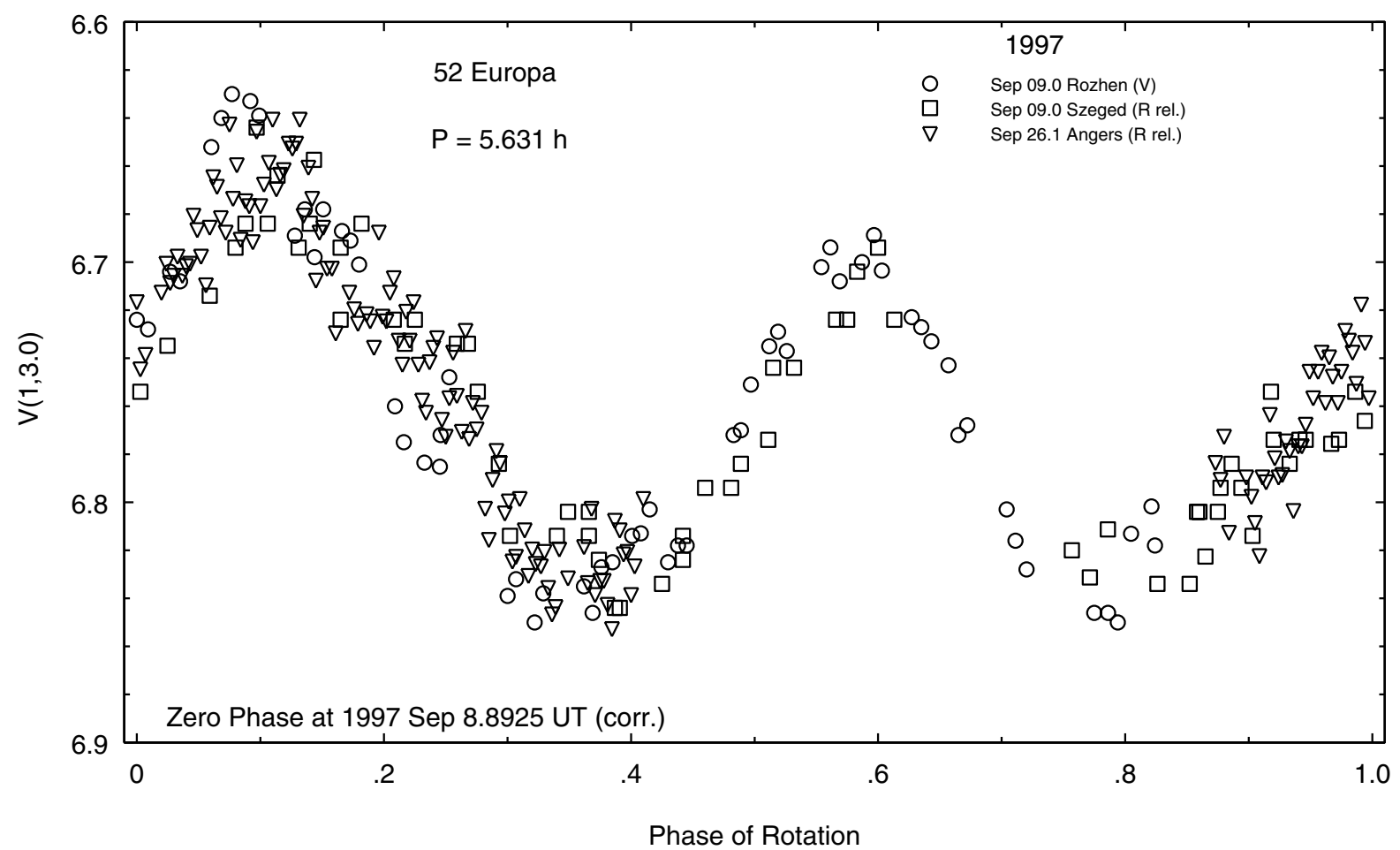

Fig. 2. Lightcurve of 52 Europa in 1997.

by Lopez-Gonzalez \& Rodriguez (1999) during the same apparition.

Our observations performed on seven nights in February-March 1997 (Fig. 3) gave the asymmetrical lightcurve with a $0.11 \mathrm{mag}$ amplitude. Only one maximum was clearly visible. However, the part of the lightcurve where the second maximum was expected was very noisy and it was difficult to recognize this extremum.

And finally, three nights of observations in February-April 2000 (Fig. 4) revealed the lightcurve with an 


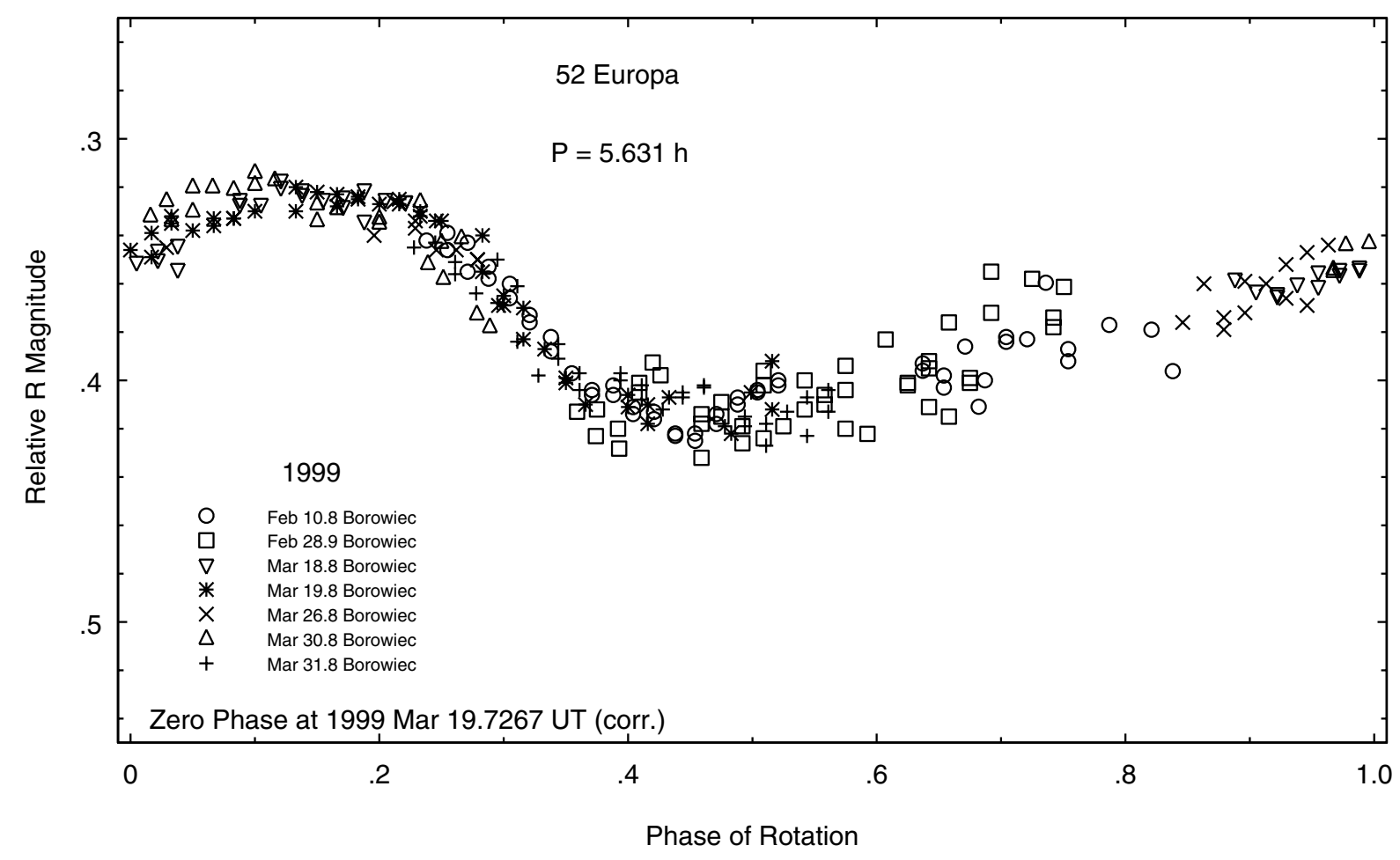

Fig. 3. Lightcurve of 52 Europa in 1999.

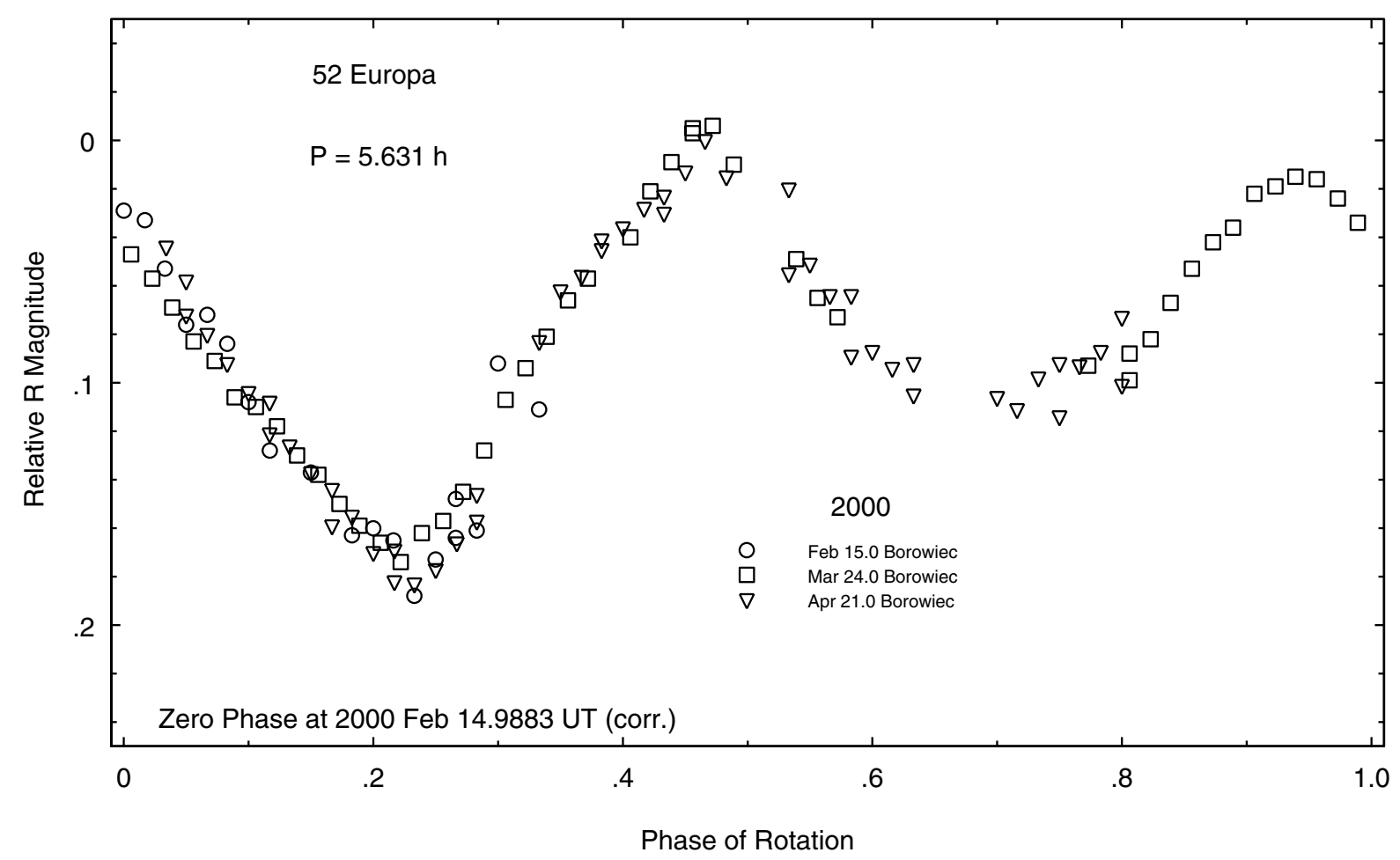

Fig. 4. Lightcurve of 52 Europa in 2000.

amplitude of $0.18 \mathrm{mag}$. This lightcurve had maxima at the similar levels and different depths in minima. It was contrary to the previous observations, when the lightcurves differed in the levels of the maxima.

\subsection{Thyra}

This asteroid was observed in 1978 by Scaltriti et al. (1981) on six nights between October 24 and December 5, and by Chang et al. (1981) on December 2 (only 3 hours run). 


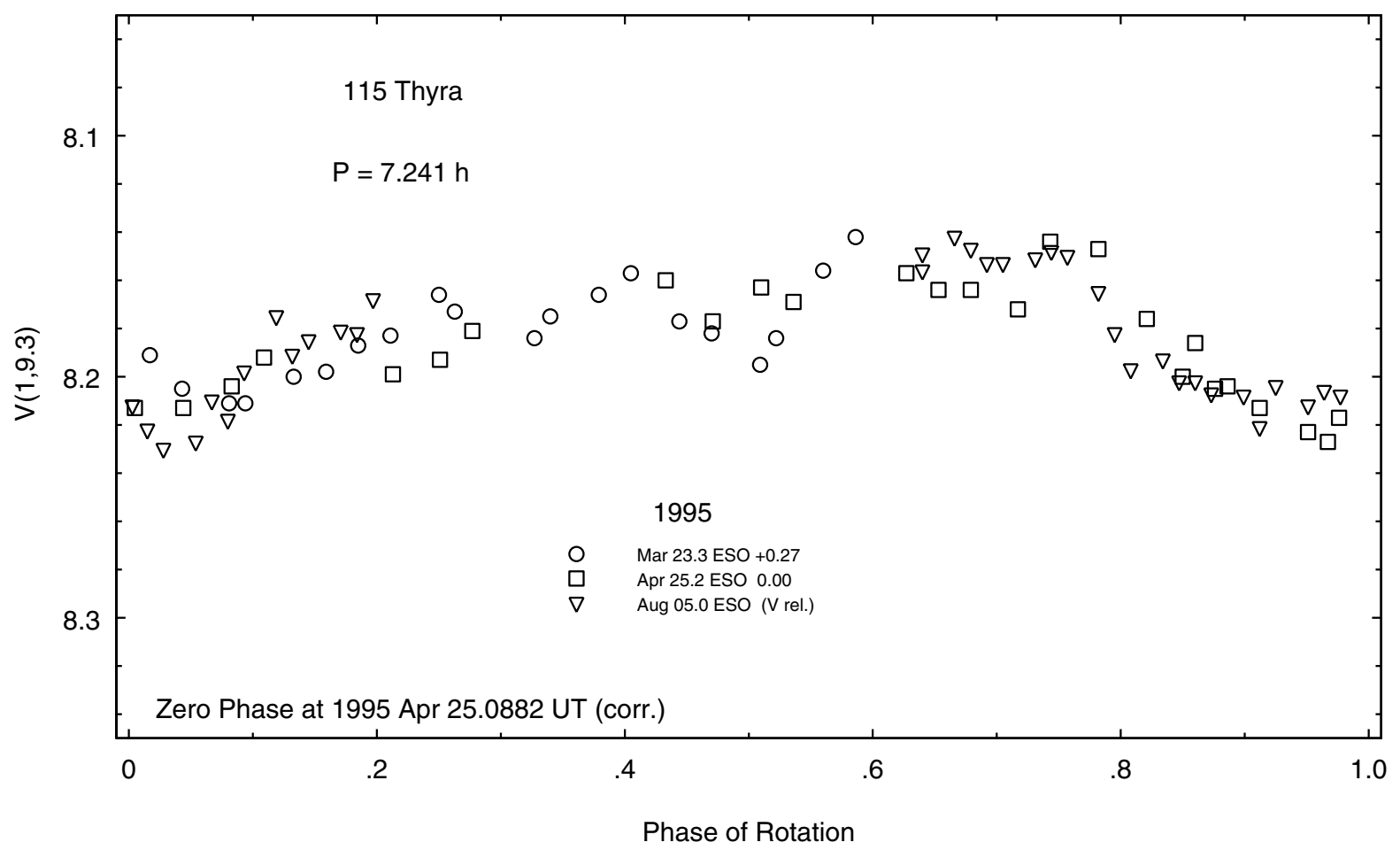

Fig. 5. Lightcurve of 115 Thyra in 1995.

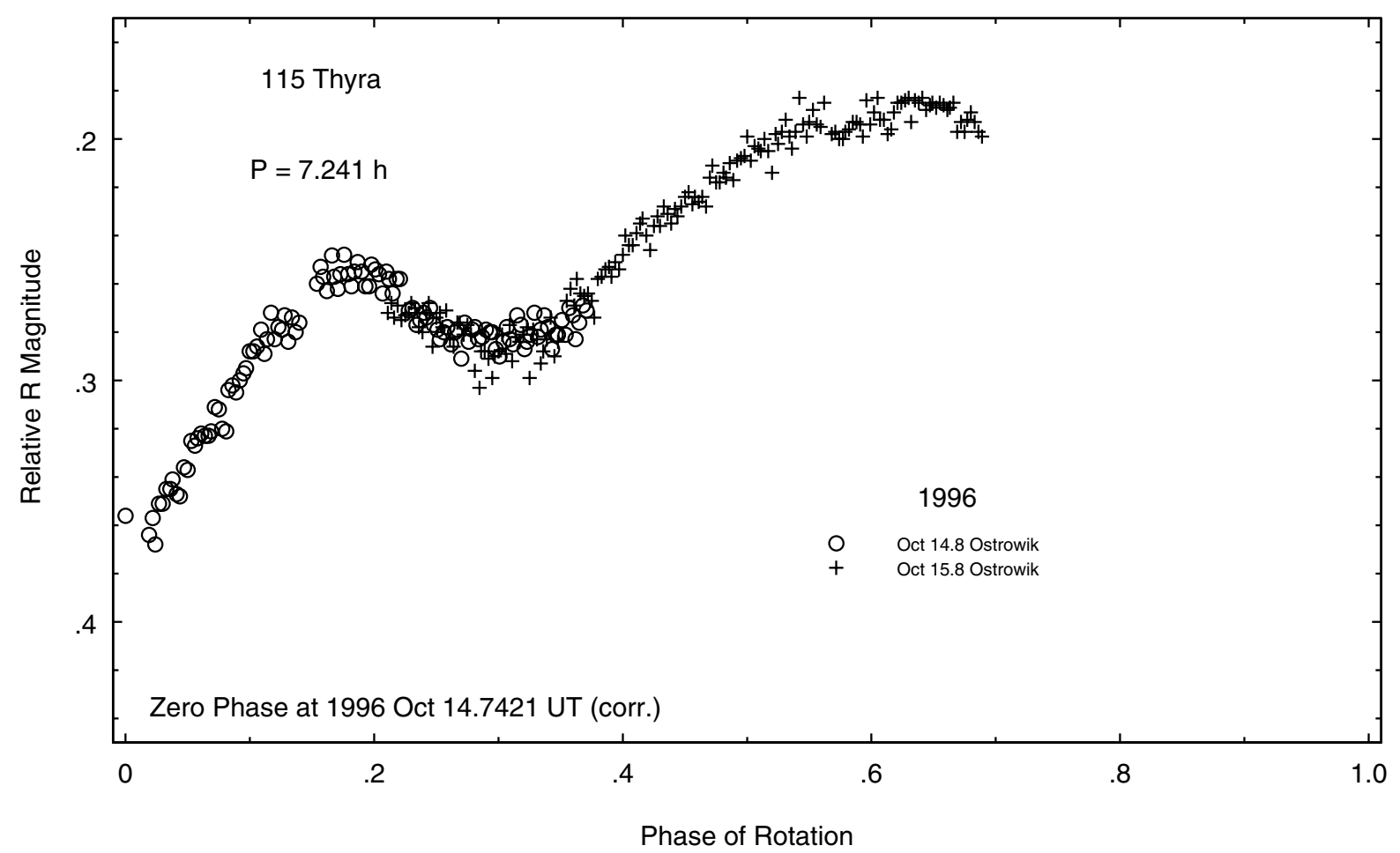

Fig. 6. Lightcurve of 115 Thyra in October 1996.

Scaltriti et al. determined a synodical period to be 7.241 hours. A composite lightcurve showed two distinct maxima and minima with an amplitude of $0.20 \mathrm{mag}$. The minima were at the same level but the maxima differed by $0.05 \mathrm{mag}$.

McCheyne et al. (1985) observed Thyra on two nights in January 1983, and obtained $B V J K$ lightcurves with amplitudes $0.13,0.13,0.15$, and $0.20 \mathrm{mag}$, respectively.
These amplitudes and colour variations would suggest that the surface of 115 Thyra had an inhomogenous composition.

In 1987 this asteroid was observed on 27 February (Magnusson \& Lagerkvist 1991) and 19, 20 March (Dotto et al. 1995). These data confirmed the rotational period of 7.241 hours but the composite lightcurve was with a smaller 


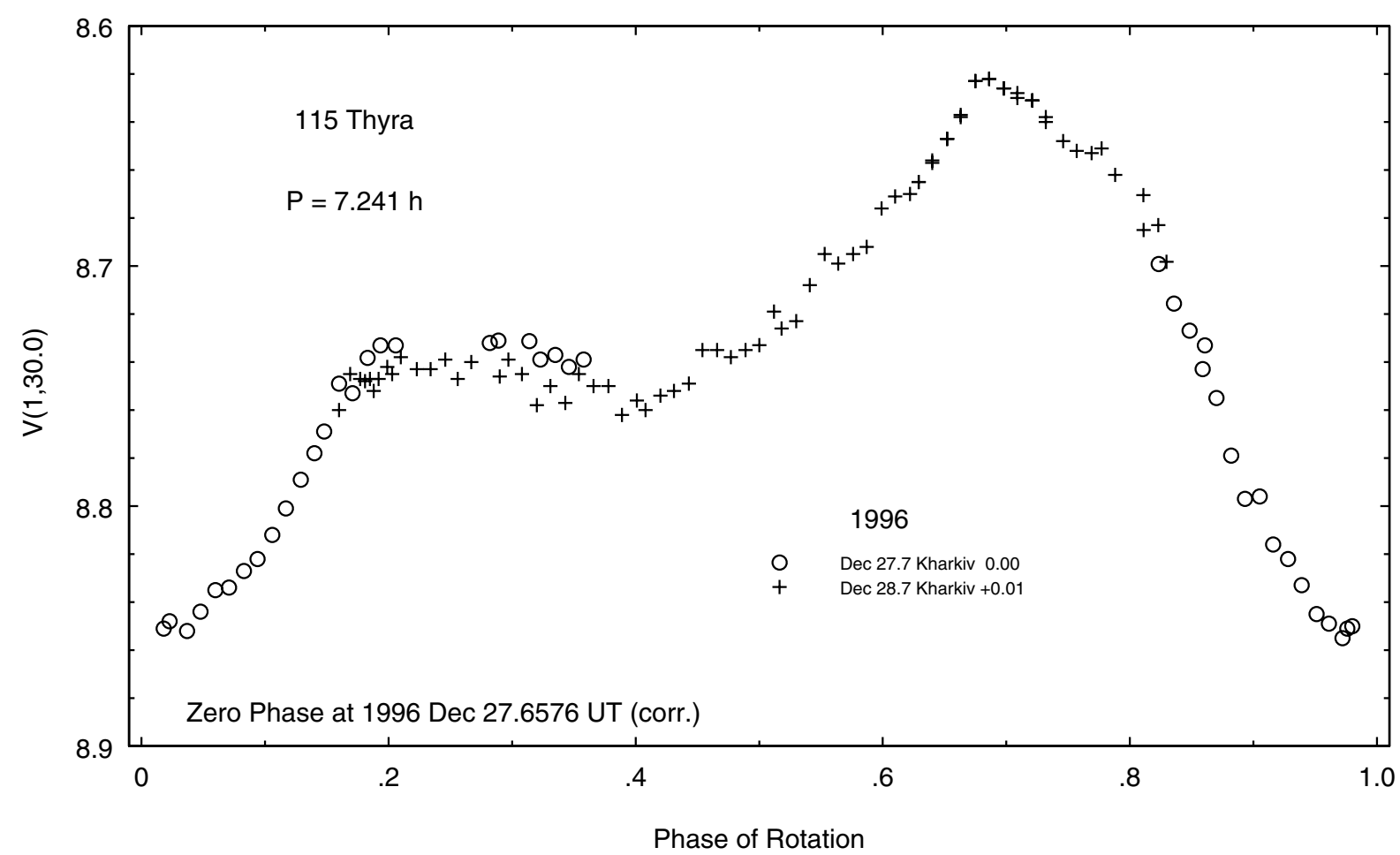

Fig. 7. Lightcurve of 115 Thyra in December 1996.

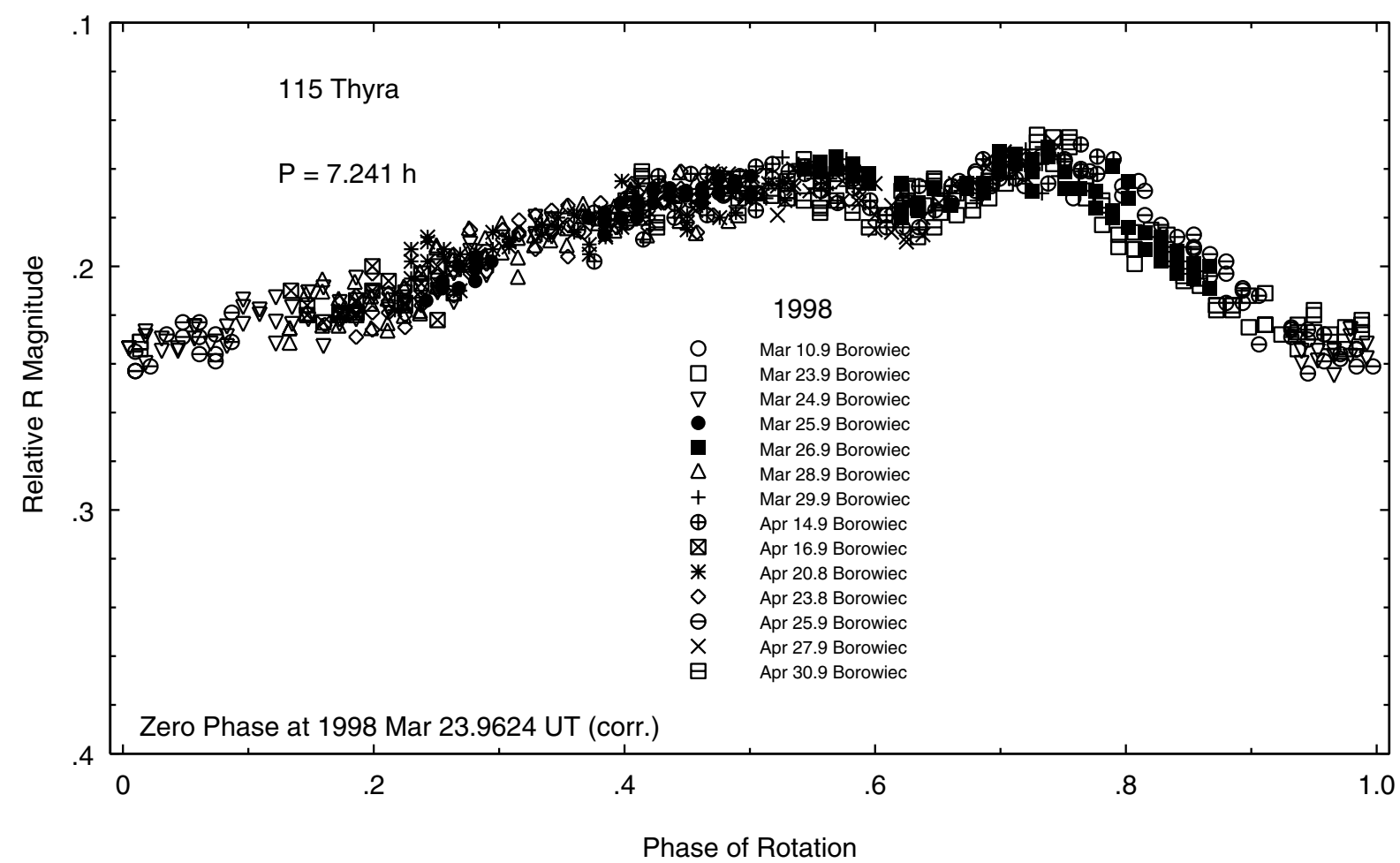

Fig. 8. Lightcurve of 115 Thyra in 1998.

amplitude of 0.08 mag and only one maximum and one minimum within this period.

During many apparitions 115 Thyra was observed with the Carlsberg Meridian Circle at La Palma in order to obtain phase relations (see Bowell et al. 1989). Lagerkvist et al. (1992) using the data from 1985, 1987, 1988, and 1989 obtained such relations with the same parameter $G$ of 0.26 . However, the observations from 1991 and 1992 apparitions (Piironen et al. 1997) gave the values for $G$ to be 0.63 and 0.40 , respectively. These values were obtained from the data covering larger range of 


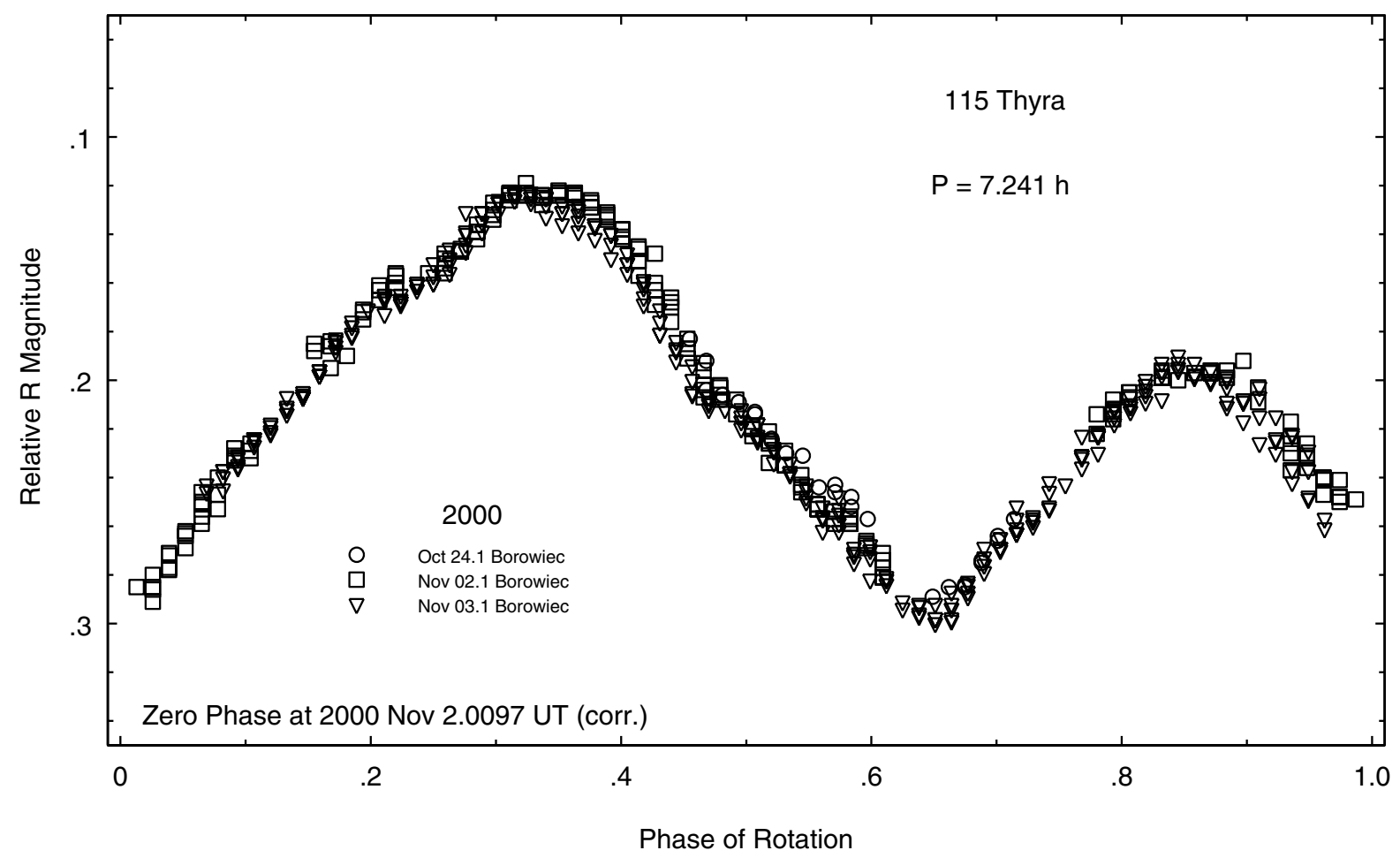

Fig. 9. Lightcurve of 115 Thyra in 2000.

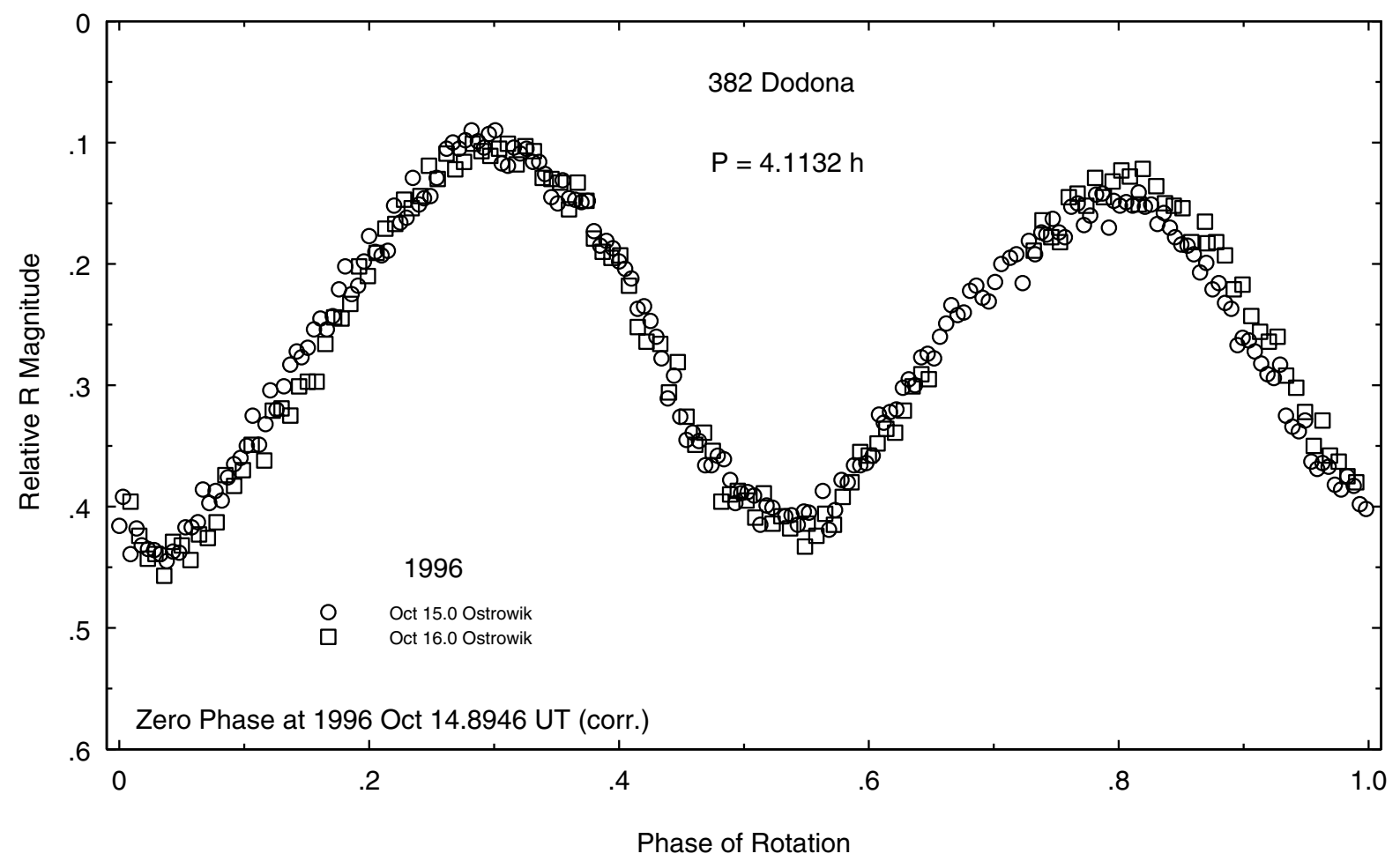

Fig. 10. Lightcurve of 382 Dodona in 1996.

phase angle (up to $27^{\circ}$ in 1992). They might confirm the remark of McCheyne et al. (1985) that Thyra had inhomogenous surface composition.

We obtained the lightcurves of 115 Thyra on 24 nights during the four apparitions: 1995, 1996, 1998, and 2000.
The observations confirmed the synodical period of 7.241 hours determined earlier.

The composite lightcurve obtained at ESO on three nights in 1995 showed a small amplitude of 0.08 mag and only one 


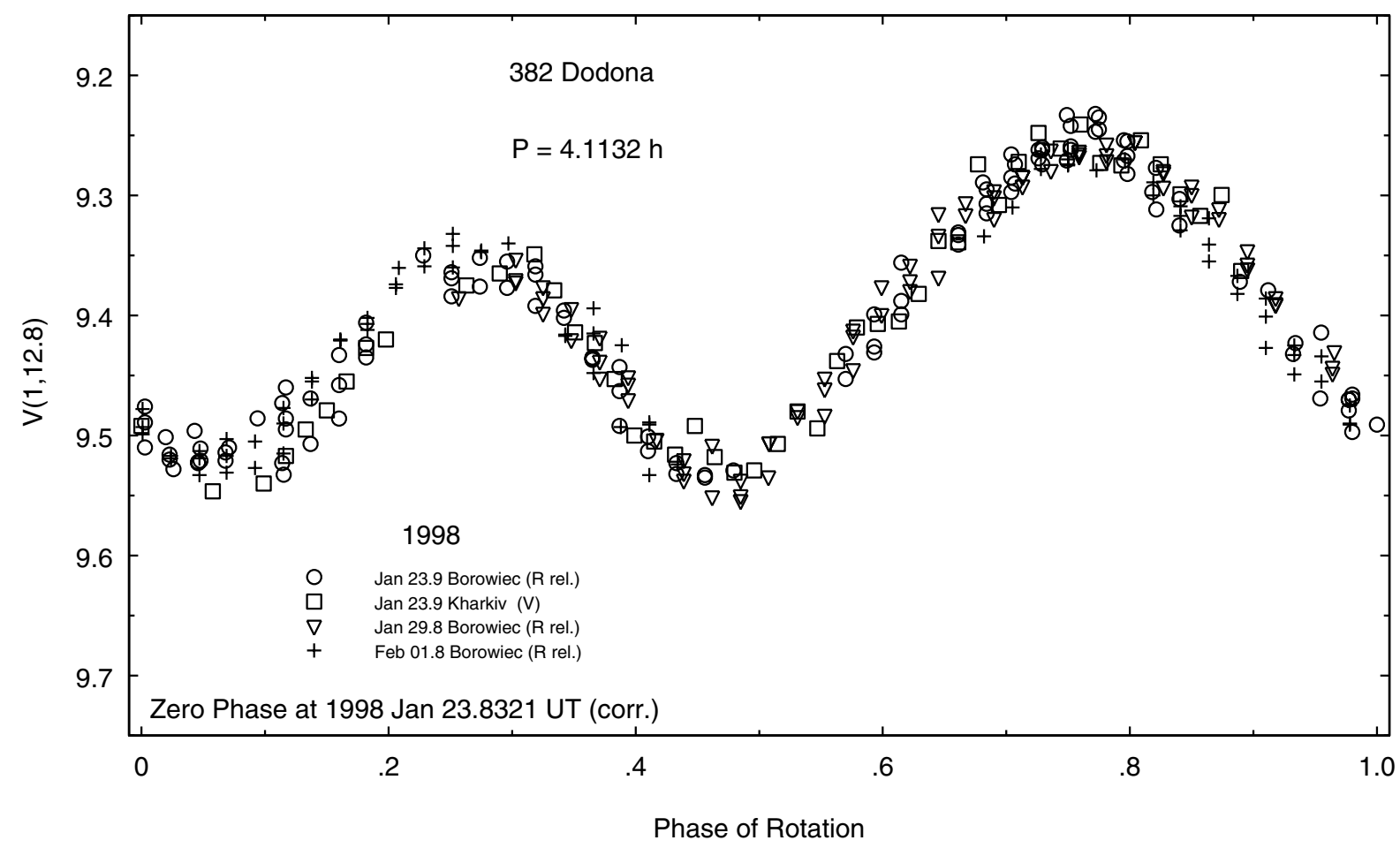

Fig. 11. Lightcurve of 382 Dodona in 1998.

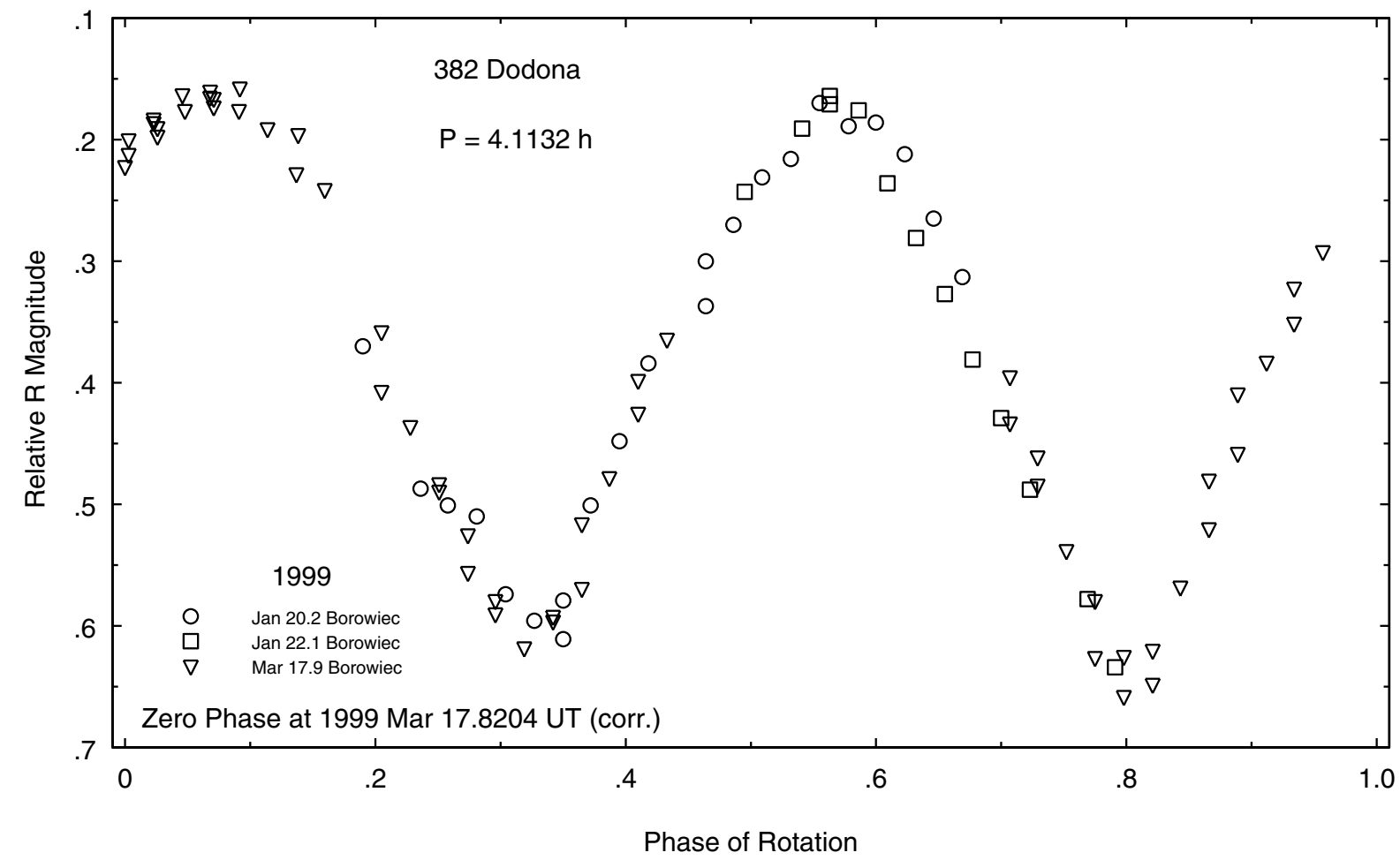

Fig. 12. Lightcurve of 382 Dodona in 1999.

pair of extrema (Fig. 5). It was similar to the observations in 1987 by Dotto et al. (1995).

Observations performed in 1996 revealed very unsymmetrical lightcurves. The data points from October (Fig. 6) covered about $70 \%$ of rotational cycle showed an amplitude of 0.17 mag. In December we obtained larger amplitude of $0.22 \mathrm{mag}$ and the shape of the lightcurve changed very much (Fig. 7). It was probably due to a large difference in the phase angle between the October and December observations $\left(20^{\circ}\right.$ and $30^{\circ}$, respectively). Because of these differences in the amplitudes and shapes we have decided to present the 1996 observations in two figures. 
In 1998 we observed Thyra on 14 nights (2.5-3 hours for each night) and obtained the composite lightcurve with the amplitude of 0.08 mag and with one maximum and one minimum (Fig. 8). The 2000 observations showed the lightcurve with the amplitude of $0.18 \mathrm{mag}$ and with one maximum lower than the other (Fig. 9).

\subsection{Dodona}

Prior to the present work this asteroid was observed during two apparitions by using a $1 \mathrm{~m}$ telescope at ESO, Chile. Lagerkvist et al. (1986) performed observations on 19 and 21 March 1983. A rotational period of 4.116 hours was derived from the brightness variation with an amplitude of 0.39 mag. Di Martino (1986) observed Dodona on 16 August 1984 and obtained a lightcurve with an amplitude of $0.42 \mathrm{mag}$.

We observed 382 Dodona on 10 nights during the four apparitions: 1996, 1998, 1999, and 2001. We were not able to make a composite lightcurve from the 1999 data when using the previously determined period of 4.116 hours. In this year, our observations span almost two months (20 Jan.-17 Mar.) covering more than 300 rotational cycles. It allowed us to determine precisely the rotational period to be of $4.1132 \pm 0.0002$ hours. The previous determination is consistent with this new one.

The lightcurves of 382 Dodona are rather regular sinusoids (Figs. 10-13) with amplitudes of 0.35, 0.29, 0.48, $0.46 \mathrm{mag}$ during those four apparitions, respectively. The observed amplitudes and their changes, indicate that Dodona is a rather elongated body with a north pole far from the Ecliptic Plane.

\section{Poles and shapes of the observed asteroids}

The spin vectors, sidreal periods, and triaxial ellipsoid models of the observed asteroids were determined by the method described by Michałowski (1993). In this method the magnitudes, amplitudes, and epochs of maxima are taken into account. The results were obtained by building a set of nonlinear equations whose solutions were found by least square fitting. The observed amplitudes and magnitudes of the brightness maxima were reduced to zero phase angle by using the amplitude-phase (Zappala et al. 1990) and the $H G$-magnitude system (Bowell et al. 1989), respectively.

As described by Michałowski (1993), the method had two-fold ambiguity in the location of an asteroid pole when the sense of rotation was fixed. This problem was also discussed earlier in a review paper by Magnusson et al. (1989). They stated that by combining the solutions from amplitudemagnitude and epoch methods, it was sometimes possible to obtain a unique solution even though each individual method had failed to achive this. However, they did not discuss when such situations were possible.

The method used in the present paper combines these methods (instead of solutions alone) in one process of calculation as mentioned above. We can also state that in some cases it is possible to obtain a unique solution for the pole location. This possibility can sometimes occur when an asteroid is observed during a few oppositions and the ecliptic latitudes are within a wide range of values (usually from about $-20^{\circ}$ to $+20^{\circ}$ ).
The second method used in the present paper is so-called lightcurve inversion as described by Kaasalainen \& Torppa (2001) and Kaasalainen et al. (2001, 2003). This method makes use of all data points (both relative and absolute photometry) and finds a physical model with a large number of parameters that accurately reproduces the photometric data down to noise level. Sample fits for the asteroids studied here are shown in Figs. 14-16. As for other methods, $\lambda_{\mathrm{p}} \pm 180^{\circ}$ ambiguity for the pole solution is always physically inevitable for asteroids moving very close to the ecliptic plane. However, the asteroid ecliptic latitudes larger than $\pm 10^{\circ}$ might already suffice to separate a unique best pole solution from the other symmetric possibilities, if the observing geometries are well covered.

Table 3 contains the spin and shape models for the asteroids studied in the present paper. This table shows the sidereal periods, the senses of rotation ( $P$ - prograde, $R$ - retrograde), the ecliptic coordinates (equinox 2000) of the north poles, and the ratios $a / b, b / c$ of triaxial ellipsoid models. The available results obtained by other authors are given for comparison. The methods, used for calculation, are also given: $E$ - epochs; $A$ amplitude; $M$ - magnitude; $L$ - lightcurve inversion.

\subsection{Europa}

Barucci et al. (1986) used the $E A$ method and the data from three oppositions $(1976,1983,1984)$ and obtained a model for this asteroid (see Table 3). The results from Michałowski (1993) - Table 3 - obtained with the same available low amplitude lightcurves, differed in $\beta_{\mathrm{p}}$ and $b / c$. The large value of $b / c$ in the latter paper seemed to be very unrealistic.

According to these results the smallest amplitude of brightness variation of Europa should be when the longitude of the asteroid was around $180^{\circ}$ or $360^{\circ}$. The observations from 1986 (Dotto et al. 1995) when the longitude of the asteroid was about $350^{\circ}$ did not confirm this prediction. The amplitude was $0.23 \mathrm{mag}$, the largest ever observed for this asteroid. Dotto et al. (1995) obtained results showing that Europa was a retrograde rotator and the larger $a / b$ and different $\lambda_{\mathrm{p}}$ (see Table 3) were due to the larger amplitude observed in 1986.

Adding the observations from two new oppositions (1992, 1994), Michałowski et al. (1995) confirmed the retrograde sense of rotation but a sidereal period they obtained was longer (Table 3).

Lopez-Gonzalez \& Rodriguez (1999) added new observations of Europa obtained in 1997. They used all available lightcurves and an EA method proposed by Michałowski \& Velichko (1990). The new results differed very much from the previous ones. The most important - the sense of rotation of Europa was prograde and the sidereal period was shorter than that reported in other papers (see Table 3 ). The big value of $b / c$ was probably caused by the fact that they did not use magnitudes for the modelling.

Recently, Kaasalainen et al. (2002) published their results for this asteroid. They used the lightcurve inversion method (mentioned also above) and obtained that Europa was a retrograde rotator which was contrary to the results by Lopez-Gonzalez \& Rodriguez (1999) - see Table 3. 


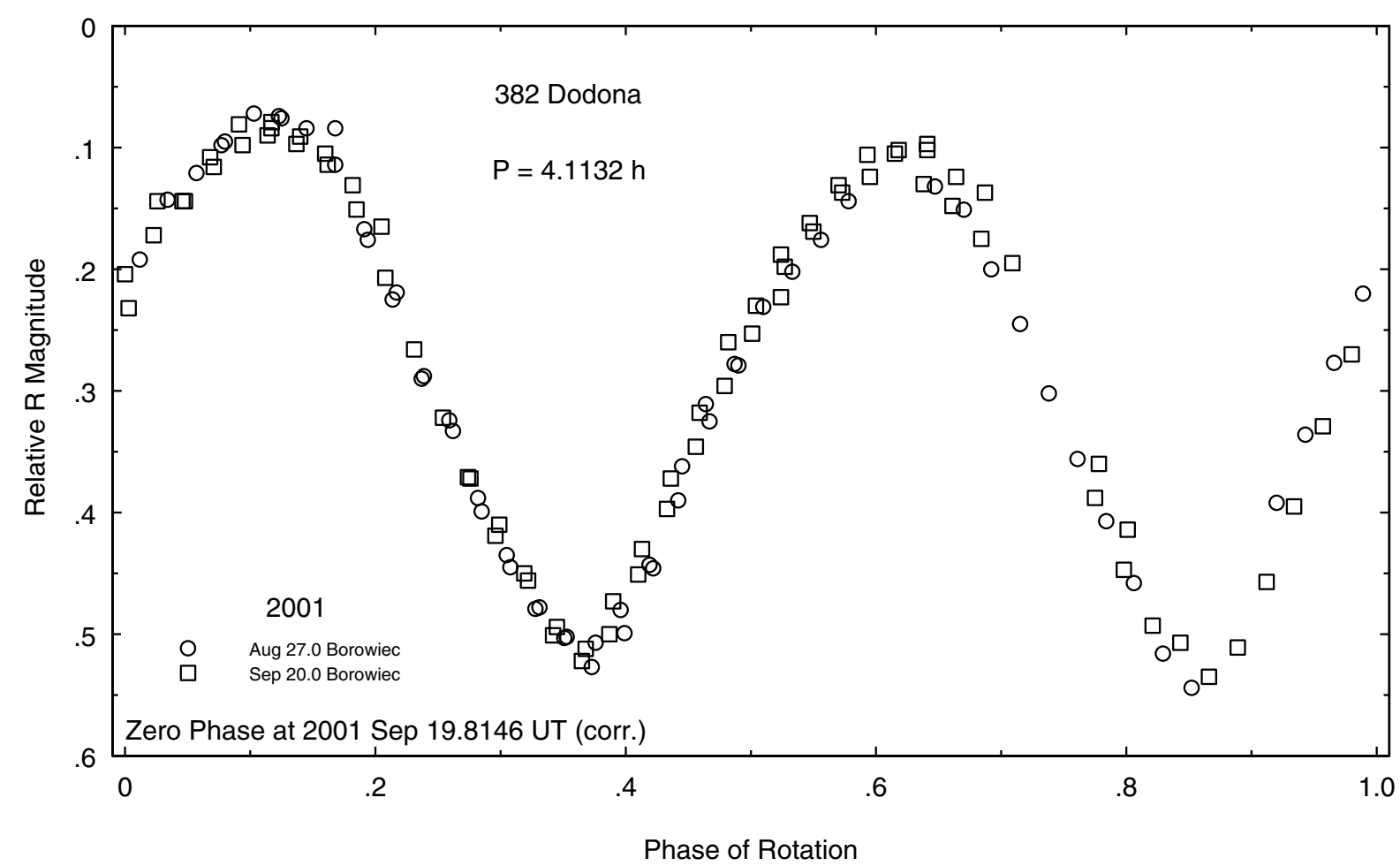

Fig. 13. Lightcurve of 382 Dodona in 2001.
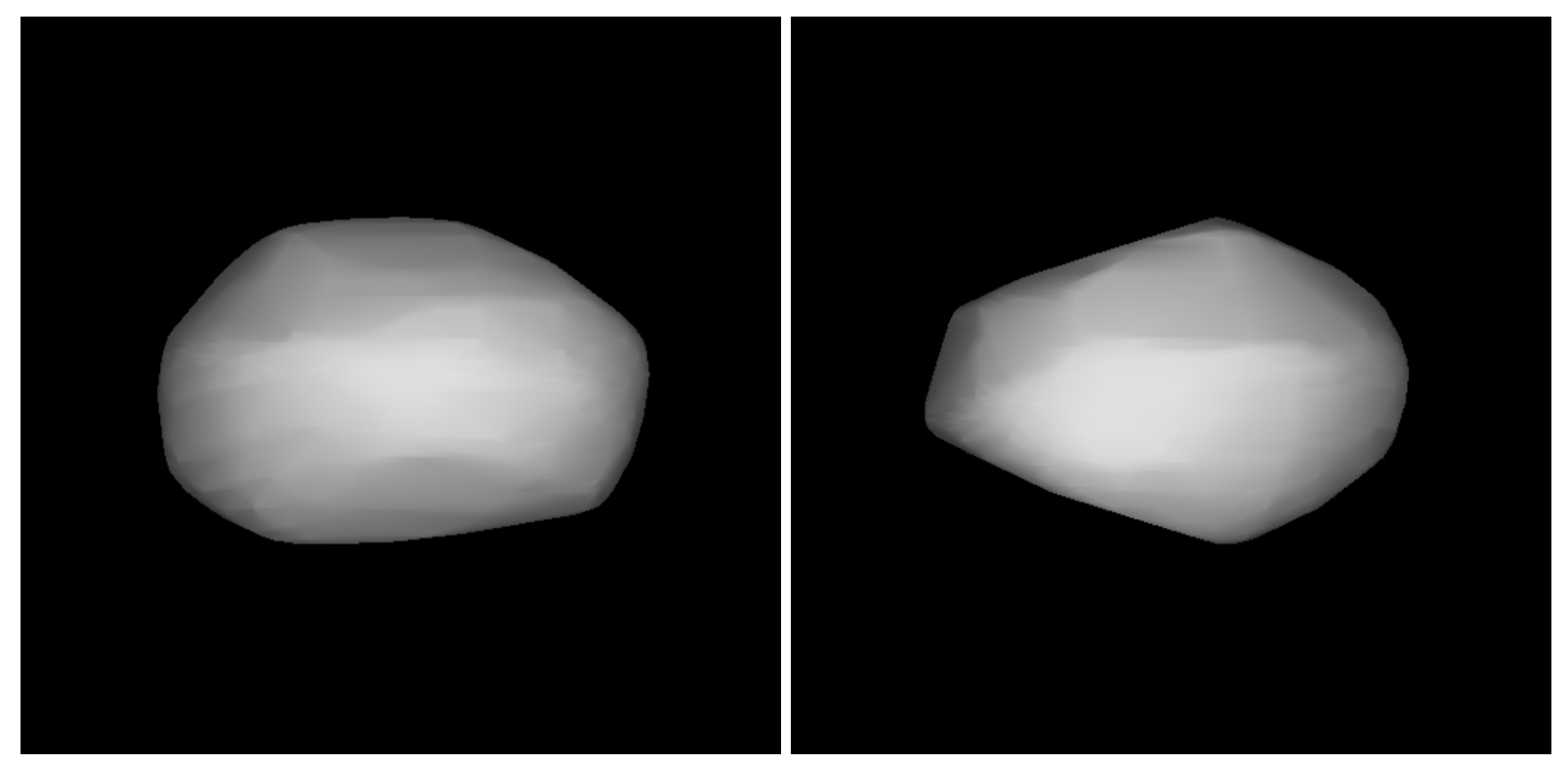

Fig. 14. Shape model of 52 Europa, shown at equatorial viewing illumination geometry, with rotational phases $90^{\circ}$ apart.

Kaasalainen et al. (2002) did not comment on this difference but they used only the observational data obtained during 6 apparitions in 1976-1994 period.

We have been able to use the lightcurves of Europa from the ten oppositions: 1976, 1983, 1984, 1986, 1992, 1994, 1995, 1997, 1999, and 2000. Our results obtained with EAM method (see Table 3) confirm the results by Lopez-Gonzalez \& Rodriguez (1999) implying that 52 Europa rotates in prograde sense.

The results obtained here with the $L$ method confirm these ones from EAM (see Table 3). Two model images $\left(90^{\circ}\right.$ apart in longitude) are in Fig. 14 and only rough relative shape dimensions are given in Table 3.

The earlier model presented in Kaasalainen et al. (2002) produced some slight discrepancies in model lightcurves, but these were thought to be due to erroneous data (or their record), and the trial period range concentrated too narrowly around the literature period estimate. With the new pole, sense of rotation and period outside that range, the earlier discrepancies disappear. Also, the new data, presented in the present work, cannot be fitted with the old period/pole solution. This case shows the 
Table 3. Spin and shape models.

\begin{tabular}{|c|c|c|c|c|c|c|c|c|c|}
\hline \multirow{2}{*}{$\begin{array}{r}\text { Sidereal } \\
\text { period (days) }\end{array}$} & \multirow{2}{*}{$\begin{array}{l}\text { Sense of } \\
\text { rotation }\end{array}$} & \multicolumn{2}{|c|}{ Pole 1} & \multicolumn{2}{|c|}{ Pole 2} & \multirow[t]{2}{*}{$a / b$} & \multirow[t]{2}{*}{$b / c$} & \multirow[t]{2}{*}{ Method } & \multirow[t]{2}{*}{ Reference } \\
\hline & & $\lambda_{p}$ & $\beta_{p}$ & $\lambda_{p}$ & $\beta_{p}$ & & & & \\
\hline \multicolumn{10}{|l|}{52 Europa } \\
\hline & & $0^{\circ}$ & $37^{\circ}$ & $203^{\circ}$ & $38^{\circ}$ & 1.12 & 1.00 & A & Barucci et al. (1986) \\
\hline & & $17^{\circ}$ & $65^{\circ}$ & & & 1.11 & 2.79 & EAM & Michałowski (1993) \\
\hline 0.2346504 & $\mathrm{R}$ & $80^{\circ}$ & $-55^{\circ}$ & $250^{\circ}$ & $-40^{\circ}$ & 1.21 & 1.30 & EA & Dotto et al. (1995) \\
\hline 0.2347019 & $\mathrm{R}$ & $84^{\circ}$ & $-32^{\circ}$ & $257^{\circ}$ & $-18^{\circ}$ & 1.20 & 1.17 & EAM & Michałowski et al. (1995) \\
\hline 0.2345855 & $\mathrm{P}$ & $261^{\circ}$ & $+60^{\circ}$ & $63^{\circ}$ & $+46^{\circ}$ & 1.19 & 2.2 & EA & Lopez-Gonzalez \& Rodriguez (1999) \\
\hline 0.2346504 & $\mathrm{R}$ & $79^{\circ}$ & $-57^{\circ}$ & $246^{\circ}$ & $-44^{\circ}$ & 1.2 & 1.2 & $\mathrm{~L}$ & Kaasalainen et al. (2002) \\
\hline 0.2345813 & $\mathrm{P}$ & $262^{\circ}$ & $+46^{\circ}$ & $71^{\circ}$ & $+31^{\circ}$ & 1.21 & 1.04 & EAM & Present work \\
\hline \pm 0.0000002 & & $\pm 9^{\circ}$ & $\pm 8^{\circ}$ & $\pm 9^{\circ}$ & $\pm 8^{\circ}$ & \pm 0.02 & \pm 0.05 & & \\
\hline 0.2345816 & $\mathrm{P}$ & $252^{\circ}$ & $+38^{\circ}$ & $67^{\circ}$ & $+25^{\circ}$ & 1.15 & 1.3 & $\mathrm{~L}$ & Present work \\
\hline \pm 0.0000002 & & $\pm 5^{\circ}$ & $\pm 5^{\circ}$ & $\pm 5^{\circ}$ & $\pm 5^{\circ}$ & & & & \\
\hline \multicolumn{10}{|l|}{115 Thyra } \\
\hline \multirow[t]{2}{*}{0.301565} & $\mathrm{P}$ & $175^{\circ}$ & $+60^{\circ}$ & $330^{\circ}$ & $60^{\circ}$ & 1.14 & 1.30 & EA & Dotto et al. (1995) \\
\hline & & $197^{\circ}$ & $30^{\circ}$ & $358^{\circ}$ & $35^{\circ}$ & 1.22 & 1.09 & $\mathrm{AM}$ & Blanco \& Riccioli (1998) \\
\hline 0.3017940 & $\mathrm{R}$ & $182^{\circ}$ & $-43^{\circ}$ & & & 1.21 & 1.03 & EAM & Present work \\
\hline \pm 0.0000003 & & $\pm 7^{\circ}$ & $\pm 7^{\circ}$ & & & \pm 0.02 & \pm 0.03 & & \\
\hline 0.3017257 & $\mathrm{P}$ & & & $7^{\circ}$ & $34^{\circ}$ & 1.23 & 1.03 & EAM & Present work \\
\hline \pm 0.0000003 & & & & $\pm 7^{\circ}$ & $\pm 7^{\circ}$ & \pm 0.02 & \pm 0.03 & & \\
\hline 0.3016652 & $\mathrm{P}$ & & & $23^{\circ}$ & $+33^{\circ}$ & 1.1 & 1.1 & $\mathrm{~L}$ & Present work \\
\hline \pm 0.0000002 & & & & $\pm 8^{\circ}$ & $\pm 4^{\circ}$ & & & & \\
\hline \multicolumn{10}{|l|}{382 Dodona } \\
\hline 0.17138450 & $\mathrm{P}$ & $88^{\circ}$ & $+68^{\circ}$ & & & 1.54 & 1.33 & EAM & Present work \\
\hline \pm 0.00000007 & & $\pm 4^{\circ}$ & $\pm 4^{\circ}$ & & & \pm 0.01 & \pm 0.07 & & \\
\hline 0.17138442 & $\mathrm{P}$ & $83^{\circ}$ & $+64^{\circ}$ & $248^{\circ}$ & $+55^{\circ}$ & 1.4 & 1.3 & $\mathrm{~L}$ & Present work \\
\hline \pm 0.00000004 & & $\pm 5^{\circ}$ & $\pm 5^{\circ}$ & $\pm 5^{\circ}$ & $\pm 5^{\circ}$ & & & & \\
\hline
\end{tabular}

importance of additional data in revising/confirming preliminary models with less than about 20 lightcurves.

\subsection{Thyra}

Dotto et al. (1995) using the lightcurves from the three oppositions $(1978,1983,1987)$ obtained prograde sense of rotation and a rough estimate of the shape, the pole coordinates, and the sidereal period (see Table 3 ). As the pole solutions were charged with huge values of formal errors $\left(20^{\circ}\right)$ they concluded that the spin and shape parameters could be different. Blanco \& Riccioli (1998) used the same observational data and the AM-method, obtained similar results (Table 3).

We have been able to use the lightcurves of Thyra obtained during the seven apparitions: 1978, 1983, 1987, 1995, 1996, 1998 , and 2000. Using the EAM method, we have obtained solutions for both retrograde and prograde senses of rotation. Pole coordinates for these two solutions indicate the same axis of rotation but for different sense of rotation and different sidereal periods (see Table 3).

The results obtained with $L$ method are given in Table 3 . As the ecliptic latitudes are not small, we got a unique pole, clearly better than any other candidates. However, Thyra seems to be a puzzling target. All models obtained with LommelSeeliger/Lambert combination or with Hapke parameters of average S-type asteroid (Helfenstein \& Veverka 1989) were somewhat too prolate, with $b / c<1$. We conclude that the lightscattering behaviour is probably somewhat different from typical scattering models. For example, we could obtain a realistic shape model by using strange Hapke parameters $S(0)=1.3$ and $g=0.3$ (insted of the more typical $S(0)=0.97$ and $g=-0.35$ for S-type). This shape is shown in the Fig. 15 . There is, of course, not enough absolute photometry (only 7 lightcurves) for obtaining any estimate of the right scattering values. Two different scattering parameter sets (and the resulting models) give exellent lightcurve fits in both relative and absolute photometry. The shape models look similar when they are squeezed or stretched to the same vertical lenght. This case thus underlines the fact that the vertical scale factor $(b / c$ ratio) of a model is never very strongly constrained using photometry alone, as discussed in, e.g., Kaasalainen et al. (2002) and Torppa et al. (2003). There are also signs of moderate albedo variegation on the surface, so some of the shape features seen in the figure can probably be partly attributed to albedo effects.

Comparing all results obtained for this asteroid (Table 3) we can conclude that 115 Thyra is a rather unusual object and 

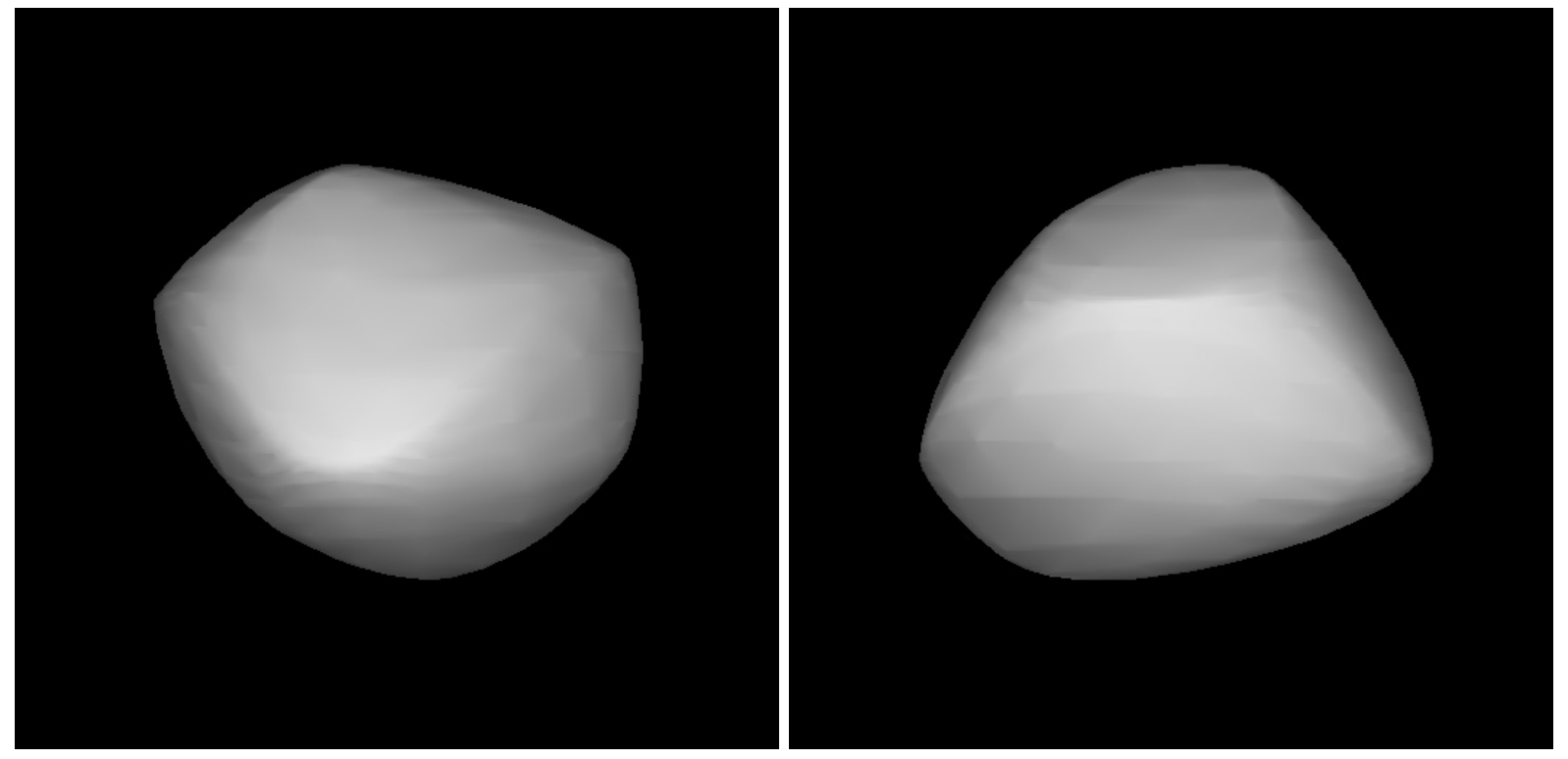

Fig. 15. Shape model of 115 Thyra.
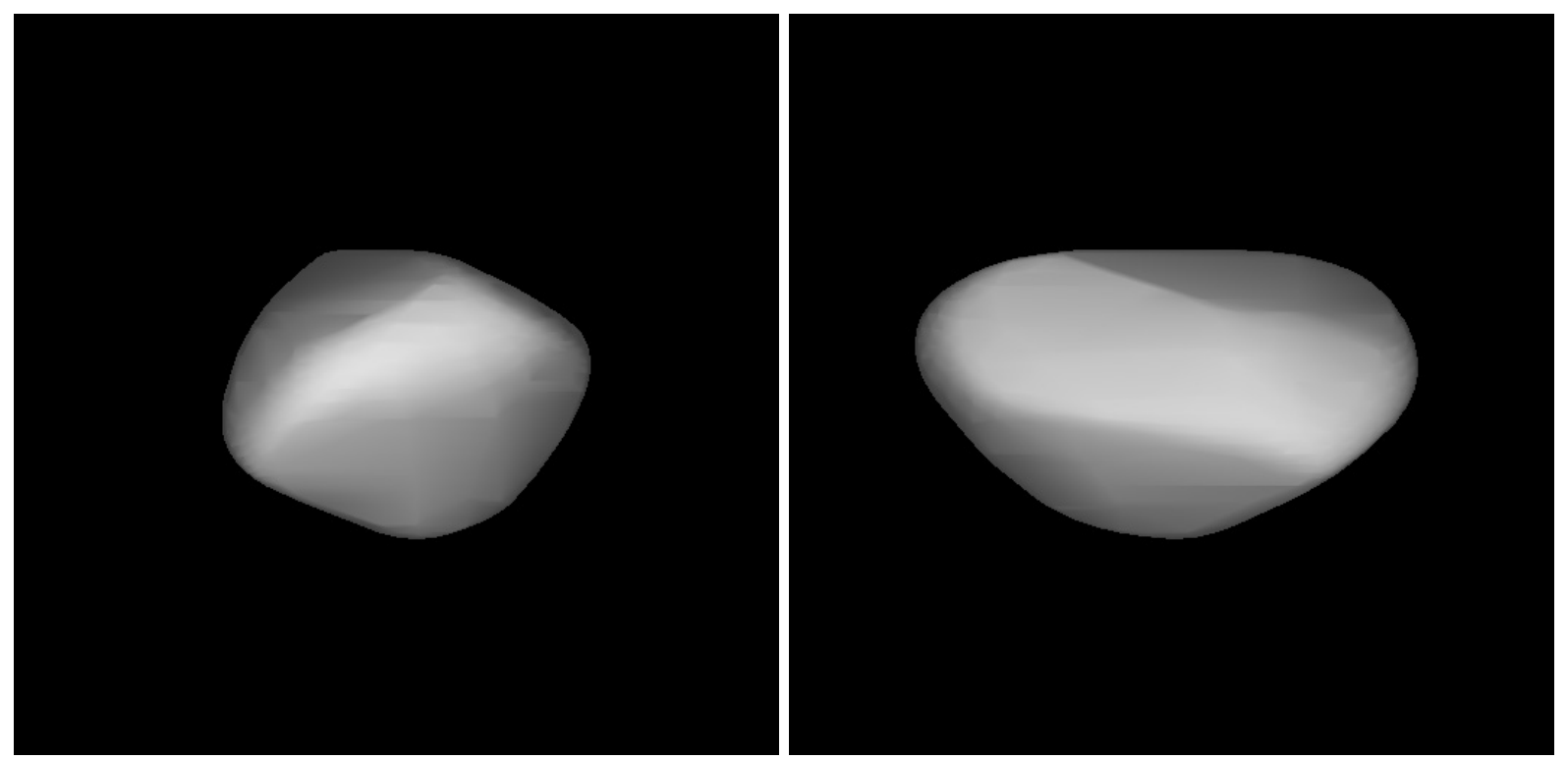

Fig. 16. Shape model of 382 Dodona.

similar to the case of 52 Europa (see above). The values of the sidereal period obtained with different methods are different and we think that new future observations will help us to resolve this discrepancy.

\subsection{Dodona}

Prior to the present paper there have been no model solutions for this asteroid.

We have used the lightcurves from the six apparitions: 1983, 1984, 1996, 1998, 1999, and 2001. The lightcurves of Dodona are very regular and close to sinusoidal shape and we have obtained similar solutions with both methods (see Table 3 ). We prefer the first $L$ pole as it gives a slightly better fit and the shape model is presented in Fig. 16.
Acknowledgements. FPV is gratefull to the DLR Institute of Planetary Exploration (Berlin, Germany) for the provision of the ST-6UV CCD camera and the image reduction software (ASTPHOT). SF thanks Alexandre Renou (Assocation Astronomique d'Anjou) for the assistance during his observations. This work was partially supported by the Polish KBN Grant 2 P03D 007 18. Support for PAD came from KBN Grant 2 P03D 007 22. The Borowiec observations were reduced with the CCLRS STARLINK package.

\section{References}

Barucci, M. A., Bockelee-Morvan, D., Brahic, A., et al. 1986, A\&A, 163,261

Blanco, C., \& Riccioli, D. 1998, A\&AS 131, 385

Bowell, E., Hapke, B., Bomingue, D., et al. 1989, in Asteroids II, ed. R. P. Binzel, T. Gehrels, \& M. S. Matthews (Univ. Arizona Press), 524 
Chang, Y. C., Zhou, X.-h., Yang, X.-y., et al. 1981, Chin. Astron. Astrophys., 5, 434

Denchev, P., Magnusson, P., \& Donchev, Z. 1998, Planet. Space Sci., 46,673

Di Martino, M. 1986, in Asteroids, Comets, Meteors II, ed. C.-I. Lagerkvist, B. A. Lindblad, H. Lundstedt, \& H. Rickman (Uppsala), 81

Dotto, E., De Angelis, G., Di Martino, M., et al. 1995, Icarus, 117, 313

Erikson, A., Mottola, S., Lagerros, J. S. V., et al. 2000, Icarus, 147, 487

Harris, A. W., \& Lupishko, D. P. 1989, in Asteroids II, ed. R. P. Binzel, T. Gehrels, \& M. S. Matthews (Univ. Arizona Press), 39

Helfenstein, P., \& Veverka, J. 1989, in Asteroids II, ed. R. P. Binzel, T. Gehrels, \& M. S. Matthews (Univ. Arizona Press), 557

Henden, A. A. 2000, J. Am. Assoc. Var. Star Obs., 29, 35

Kaasalainen, M., \& Torppa, J. 2001, Icarus, 153, 24

Kaasalainen, M., Torrpa, J., \& Muinonen, K. 2001, Icarus, 153, 37

Kaasalainen, M., Torppa, J., \& Piironen, J. 2002, Icarus, 159, 369

Kaasalainen, M., Mottola, S., \& Fulchignoni, M. 2003, in Asteroids III, ed. W. F. Bottke A. Cellino, P. Paolicchi, \& R. P. Binzel (Univ. Arizona Press), 139

Lagerkvist, C.-I., Hahn, G., Manusson, P., et al. 1986, in Asteroids, Comets, Meteors II, ed. C.-I. Lagerkvist B. A. Lindblad, H. Lundstedt, \& H. Rickman (Uppsala), 67

Lagerkvist, C.-I. Magnusson, P., Williams, I. P., et al. 1992, A\&AS, 94,43
Lopez-Gonzales, M. J., \& Rodriguez, E. 1999, A\&AS, 139, 565

Magnusson, P., \& Lagerkvist, C.-I. 1990, A\&AS, 86, 45

Magnusson, P., \& Lagerkvist, C.-I. 1991, A\&AS, 87, 269

Magnusson, P., Barucci, M. A., Drummond, J. D., et al. 1989, in Asteroids II, ed. R. P. Binzel, T. Gehrels, \& M. S. Matthews (Univ. Arizona Press), 66

McCheyne, R. S., Eaton, N., \& Meadows, A. J. 1985, Icarus, 61, 443

Michałowski, T. 1993, Icarus, 106, 563

Michałowski, T., \& Velichko, F. P. 1990, Acta Astron., 40, 321

Michałowski, T., Velichko, F. P., Di Martino, M., et al. 1995, Icarus, 118,292

Mottola, S., De Angelis, G., Di Martino, S., et al. 1995, Icarus, 117, 62

Paolicchi, P., Burns, J. A., \& Weidenschilling, S. J. 2003, in Asteroids III, ed. W. F. Bottke, A. Cellino, P. Paolicchi, \& R. P. Binzel (Univ. Arizona Press), 517

Piironen, J., Magnusson, P., Lagerkvist, C.-I., et al. 1997, A\&AS, 121, 489

Scaltriti, F., \& Zappala, V. 1997, A\&AS, 30, 169

Scaltriti, F., Zappala, V., \& Harris, A. W. 1981, Icarus, 46, 275

Szabò, Gy. M., Csàk, B., Sàrneczky, K., \& Kiss, L. L. 2001, A\&A, 375,285

Torppa, J., Kaasalainen, M., Michałowski, T., et al. 2003, Icarus, 164, 346

Udalski, A., \& Pych, W. 1992, Acta Astron., 42, 285

Zappala, V., Di Martino, M., \& Cacciatori, S. 1983, Icarus, 56, 319

Zappala, V., Cellino, A., Barucci, M. A., et al. 1990, A\&A, 231, 548 